\title{
Management of the early postoperative PCR positive patients in the COVID-19 pandemic: cardiac surgeon's nightmare
}

\author{
Ahmet Kağan Asø, Şenol Yavuz® \\ Department of Cardiovascular Surgery, University of Health Sciences, Bursa Medical Faculty, Bursa Yüksek Ihtisas Training and \\ Research Hospital, Bursa, Turkey
}

\begin{abstract}
Objectives: In the last 2 years with the new type of coronavirus infection (COVID-19) pandemic, it has become inevitable to adapt to this disease in cardiovascular surgery procedures. In this study, we aimed to investigate the effects of the results of respiratory tract samples taken from different places in patients undergoing cardiac surgery on our postoperative patient follow-up procedures and to share our cardiac surgery experiences during the pandemic period.

Methods: A total of 177 patients who underwent cardiac surgery were included in this study. Endobronchial lavage samples were obtained from the intubated patients through the endotracheal tube in the early postoperative period. According to the Reverse Transcriptase Polymerase Chain Reaction (RT-PCR) results obtained in the early postoperative period, the patients were divided into 2 groups as Group 1 for those with negative PCR and Group 2 for those with positive PCR. After that a total of 59 patients who were found to have COVID-19 were divided into 2 groups as survivors and non-survivors.

Results: There were $118(66.6 \%)$ patients in Group 1 and $59(33.3 \%)$ in Group 2. The mean ages of patients in Group 1 and Group 2 were $64.5 \pm 9.8$ years and $61.9 \pm 10.1$ years, respectively $(p=0.174)$. Mortality was significantly higher in Group $2(\mathrm{n}=24,40.6 \%)$ compared Group $1(\mathrm{n}=2,1.6 \%)(p<0.001)$. After that a total of 59 patients who were found to have COVID-19 were divided into 2 groups as survivors $(\mathrm{n}=35,59.3 \%)$ and non-survivors $(n=24,40.7 \%)$. There was no statistically significant difference between the groups in terms of gender, smoking, diabetes mellitus, hypertension, chronic renal failure, chronic obstructive pulmonary diseas rates and surgery types $(p>0.05)$.

Conclusions: The COVID-19 pandemic has significantly affected our cardiovascular surgery practice. In addition to being negative for PCR at least 2 times in routine preoperative preparations, obtaining endobronchial lavage samples for PCR testing from the endotracheal tube in the early postoperative period plays an important role in patient management.
\end{abstract}

Keywords: COVID-19, cardiac surgery, endobronchial lavage, nasopharyngeal swab, mortality

$\mathrm{T}$ he new type of coronavirus infection (COVID19), which emerged in the Wuhan province of China in December 2019, spread very rapidly and affected the whole world. On March 11, 2020, the World
Health Organization (WHO) declared that this disease caused a pandemic. In the last 2 years, the world has developed new strategies to combat this disease and has entered into a restructuring in terms of approach 
to the disease.

As in all medical departments, it has become inevitable to adapt to this new situation in cardiovascular surgery procedures. For this purpose, a series of precautions have been taken for cardiac patients, who are reported as one of the most risky groups. First of all, postponing elective cardiac surgical procedures, applying less invasive procedures if the operation cannot be postponed, and performing the operation within a certain algorithm and taking the high surgical risk into account, if the operation is inevitable, constituted the main approach scheme [1-3]. It has been reported that pulmonary complications related to COVID-19, especially in the postoperative period, have very serious consequences for this critically ill population [4-6]. In order to avoid these fatal outcomes, it is recommended to plan the operation by seeing 2 negative Reverse Transcriptase Polymerase Chain Reaction (RT-PCR) results in the preoperative period [7]. Although the reliability of samples taken by nasopharygeal swab (NFS) is high, it has been shown that the positivity rate is different compared to samples taken from the lower respiratory tract [8]. The COVID-19 pandemic had negative effects on the number of surgical procedures and postoperative morbidity and morbidity rates in our cardiovascular surgery clinic.

The aim of this study is to share our experience in patients who underwent cardiac surgery in our heart center, which has been active since the beginning of the pandemic, and to reveal how endobronchial lavage samples taken from the endotracheal tube in the early postoperative period have changed our postoperative patient management procedures.

\section{METHODS}

\section{Data Sources}

For this study, approval was obtained from the T.C. Ministry of Health General Directorate of Health Services COVID-19 Scientific Research Evaluation Commission and Bursa Yüksek Ihtisas Training and Research Hospital Clinical Research Ethics Committee with the protocol number 2011-KAEK-25 2021/06-06. In this study, which was planned as a single center, patients aged between 38-91 years who underwent open heart surgery in Bursa Yüksek Ihtisas Training and Research Hospital Cardiovascular Sur- gery clinic between $01 / 09 / 2020$ and $31 / 12 / 2021$ were examined. The laboratory values, radiological image data, demographic characteristics, measured vital parameters, applied treatments and clinical outcomes of the patients were recorded at the time of admission to the hospital. According to our hospital protocol, thoracic computed tomography (CT) has been routinely performed in all patients scheduled for open heart surgery since the beginning of the COVID-19 pandemic. For this reason, routine thoracic CT was performed in all patients included in the study. Patients with positive RT-PCR in the preoperative period, patients with pathological images in radiological imaging, emergency operations, redo operations, previous lung malignancy or pneumonia history, pre-operative diagnosis of COVID-19, and patients less than 1 month from the diagnosis of COVID-19 were not included in the study. Patients who were found to have negative RT-PCR test in two consecutive nasopharyngeal swab samples taken from the nasopharyngeal region in the last 48 hours in the preoperative period were included in the study. In addition, endobronchial lavage from the intubation tube and RT-PCR test were performed in all patients within the first 15 minutes postoperatively. As a result, a total of 548 patients were examined. According to the RT-PCR results obtained in the postoperative period, the patients were divided into 2 groups as Group 1 for PCR negative and Group 2 for PCR positive. While there were 59 patients in Group 2, the sample group was formed with 118 patients by determining the ratio of $2: 1$ through the computer program among the remaining 489 patients for Group 1.

\section{Variables}

Variables were recorded from the time of patients' first admission to the hospital. Demographic data, age, gender, and smoking were recorded. Hypertension (HT), diabetes mellitus (DM), chronic obstructive pulmonary disease (COPD), coronary artery disease (CAD), and chronic renal failure (CRF) were recorded in their medical history. Two consecutive RT-PCR results obtained with NFS in the last 48 hours preoperatively, and RT-PCR results obtained from the endobronchial lavage sample taken from the endotracheal intubation tube and examined within the first 15 minutes after the patient's transfer to the intensive care unit in the postoperative period were recorded. 


\section{Statistical Analysis}

In our study, SPSS version 21.0 (IBM Statistical Package for the Social Sciences Statistic Inc., Chicago, IL, USA) program was utilized to analyze the data. "Kolmogorov-Smirnov test and ShapiroWilk test" were used for normality distribution analysis. Student's t test was used for the data presenting normal distribution and Mann-Whitney $U$ test for those that did not conform to normal distribution. These data were shown as mean \pm standard deviation or as mean (interquartile range). Categorical variables were shown as frequency and percentage, and "chisquare test" was used for analysis. A $p<0.05$ was accepted statistically significant.

\section{RESULTS}

Endobronchial lavage samples were obtained from the endotracheal tube in the early postoperative period in 177 patients included in the study. There were 118 (66.6\%) patients in Group 1 and 59 (33.3\%) in Group 2. The mean ages of patients in Group 1 and Group 2 were $64.5 \pm 9.8$ years and $61.9 \pm 10.1$ years, respectively $(p=0.174)$. There was no statistically significant difference between the groups in terms of gender, smoking, DM, HT, CRF, COPD rates and surgery types $(p>0.05$ for all). Mortality was significantly higher in Group $2(\mathrm{n}=24,40.6 \%)$ compared Group 1 $(\mathrm{n}=2,1.6 \%)(p<0.001)$ (Table 1).

A total of 59 patients who were found to have COVID-19 were divided into 2 groups as as survivors $(\mathrm{n}=35,59.3 \%)$ and non-survivors $(\mathrm{n}=24,40.7 \%)$. The mean ages of patients in survivor and non-survivor were $60.4 \pm 10.3$ years and $64.1 \pm 9.9$ years, respectively $(\mathrm{p}=0.239)$. There was no statistically significant difference between the groups in terms of gender, smoking, DM, HT, CRF, COPD rates and surgery types $(p>0.05$ for all).

\section{DISCUSSION}

In this study, we found that in addition to our cardiac surgery experience during the COVID-19 pandemic period, the way RT-PCR was performed creates significant differences in patients who were planned and performed cardiac surgery. The results of endobronchial lavage samples taken from the intubation tube in the early postoperative period have led us to take vitally important decisions in patient and disease management after cardiac surgery. By virtue of the algorithm we developed, we applied a multi-disciplinary approach to this special patient group by taking isolation measures in the postoperative period. Thus, PCR positive patients are isolated from other patients, min-

Table 1. Demographic and peroperative features of the patients

\begin{tabular}{|c|c|c|c|}
\hline Variables & $\begin{array}{c}\text { Group 1 } \\
\text { (PCR negative) } \\
(\mathbf{n}=\mathbf{1 1 8})\end{array}$ & $\begin{array}{c}\text { Group } 2 \\
\text { (PCR positive) } \\
(n=59)\end{array}$ & $p$ value \\
\hline Age (years) $($ mean \pm SD) & $64.5 \pm 9.8$ & $61.9 \pm 10.1$ & 0.174 \\
\hline Male gender, $\mathrm{n}(\%)$ & $59(50)$ & $34(57.6)$ & 0.338 \\
\hline Hypertension, n (\%) & $20(16.9)$ & $14(23.7)$ & 0.280 \\
\hline Diabetes mellitus, n (\%) & $28(23.7)$ & $10(16.9)$ & 0.400 \\
\hline COPD, n (\%) & $5(4.2)$ & $6(10.1)$ & 0.123 \\
\hline Smoking, n (\%) & $24(20.3)$ & $14(27.1)$ & 0.605 \\
\hline CRF, n (\%) & $3(2.5)$ & $1(1.6)$ & 0.721 \\
\hline Surgery type, n (\%) & & & 0.345 \\
\hline Isolated CABG & $93(78.8)$ & $50(84.7)$ & --- \\
\hline Combined surgery & $25(21.2)$ & $9(15.3)$ & --- \\
\hline Mortality, n (\%) & $2(1.7)$ & $24(40.7)$ & $<0.001$ \\
\hline
\end{tabular}

$\mathrm{COPD}=$ Chronic obstructive pulmonary disease, $\mathrm{CRF}=$ Chronic renal failure, $\mathrm{SD}=$ standard deviation, $\mathrm{PCR}=\mathrm{Polymerase}$ chain reaction, $\mathrm{CABG}=$ Coronary artery bypass graft 
Table 2. Demographic and peroperative features of PCR positive patients

\begin{tabular}{lccc}
\hline Variables & $\begin{array}{c}\text { Survivor } \\
(\mathbf{n}=\mathbf{3 5})\end{array}$ & $\begin{array}{c}\text { Non-survivor } \\
(\mathbf{n}=\mathbf{2 4})\end{array}$ & $p$ value \\
\hline Age (years) (mean \pm SD) & $60.4 \pm 10.3$ & $64.1 \pm 9.9$ & 0.239 \\
\hline Male gender, $\mathbf{n}(\%)$ & $19(54.2)$ & $15(62.5)$ & 0.531 \\
\hline Hypertension, $\mathbf{n}(\%)$ & $8(22.8)$ & $6(25.0)$ & 0.849 \\
\hline Diabetes mellitus, $\mathbf{n}(\%)$ & $2(5.7)$ & $8(33.3)$ & 0.015 \\
\hline COPD, $\mathbf{n}(\%)$ & $2(5.7)$ & $4(16.6)$ & 0.174 \\
\hline Smoking, $\mathbf{n}(\%)$ & $7(20.0)$ & $7(29.1)$ & 0.416 \\
CRF, $\mathbf{n}(\%)$ & 0 & $1(4.1)$ & 0.848 \\
\hline Surgery type, $\mathbf{n}(\%)$ & & & 0.626 \\
\multicolumn{1}{c}{ Isolated CABG } & $29(82.8)$ & $21(87.5)$ & --- \\
\hline \multicolumn{1}{c}{ Combined surgery } & $6(17.2)$ & $3(12.5)$ & -- \\
\hline
\end{tabular}

$\mathrm{COPD}=$ Chronic obstructive pulmonary disease, $\mathrm{CRF}=$ Chronic renal failure, $\mathrm{CABG}=$ Coronary artery bypass graft

imizing the risk of transmission.

As of the COVID-19 pandemic, radical changes have occurred in the practice of cardiac surgery. There has been a significant decrease in the patient population undergoing cardiac surgery. Also it has emerged that elective procedures should be deferred, and interventions that cannot be deferred should be performed under strict precautions and control, especially in patients who need cardiac surgery. Cardiac surgery practices are associated with a high mortality risk during the pandemic, especially in the presence of coronary artery disease [9-11]. This situation emerges as a need for long hospital stays at the patient level, intensive care or service follow-up. For this reason, the group of patients who underwent cardiac surgery constituted a sensitive and unique population for the pandemic period.

As of the beginning of the pandemic, there has been a significant decrease in the patient population undergoing cardiac surgery. These operations were avoided due to the high data on cardiac surgical mortality associated with COVID-19. Surgical procedures were applied under strict precautions for operations that could not be postponed. In our clinic, cardiac surgery applications were performed in accordance with a certain procedure. Especially in the early postoperative period, it became necessary to develop an algorithm after PCR positivity was observed in endobronchial lavage samples taken from the intubation tube. With this algorithm, all patients were considered potential PCR positive in the early postoperative period, until the PCR tests from endobronchial lavage samples were concluded. Care was taken in both the intensive care follow-up of the patient and the personal protection equipment of the healthcare workers. Those with positive RT-PCR tests were immediately transferred to the COVID-19 intensive care unit, while those with negative RT-PCR continued to be followed up in the classic cardio-vascular intensive care unit. A multidisciplinary approach was applied to cardiac surgery patients who were positive for PCR, and their follow-up and treatment was planned with the departments of pulmonary diseases, infectious diseases, anesthesiology and cardiology.

The clinical picture of COVID-19 infection appears in a wide spectrum ranging from asymptomatic disease carrier to pneumonia with diffuse lung involvement, multi-organ failure and sepsis [12]. Although the majority of patients survive the disease with mild to moderate severity, in the study of Guan et al. [13], the rate of severe disease and hospitalization among patients was reported to be around $16 \%$. The symptoms of the disease are seen to vary from mild disease findings to severe disease. It has been reported that there are comorbidities such as CAD, HT, DM, immunodeficiency or malignancy in the patient group in which the disease progresses particularly severely $[9,14]$. There is no comprehensive study in the literature regarding postoperative mortality rates in patients with COVID-19 infection, especially in patients undergoing cardiac surgery. In studies conducted with a small number of patients, mortality rates have been 
reported to range from $8 \%$ to $50 \%[5,15,16]$. Considering the in-hospital mortality rates, the mortality rate is up to $84 \%$ in patients undergoing cardiac surgery who need intensive care again [2].

In our study, mortality rate was found to be $40.7 \%$ in RT-PCR positive COVID-19 patients detected in the early period after cardiac surgery. In the study of Lei et al. [17] performed on patients who underwent noncardiac surgery and were in the incubation period for COVID-19 infection, the total mortality rate was $20.6 \%$, while the mortality rate for the patient group requiring intensive care was reported as $46.7 \%$. In this study, it is seen that the high mortality rate detected in patients requiring intensive care follow-up after major surgery, even if they did not undergo cardiac surgery, is also in line with the data of our study.

RT-PCR from nasopharyngeal swab samples is typically used to diagnose COVID-19. In addition, blood, sputum, stool, urine and broncho-alveolar swab samples can also be examined for diagnosis. In a study conducted on 1070 different samples from 205 patients, the highest rate of RT-PCR positivity was found in endobronchial lavage samples $(93 \%)$. This is followed by sputum $(72 \%)$, nasal swab $(63 \%)$ and pharyngeal swab (32\%) [8]. As can be seen, endobronchial lavage samples taken from the intubation tube show a high RT-PCR positivity. This also means that endobronchial lavage specimen collection yields a high-confidence result compared to RT-PCR tests from nasopharyngeal swab specimens. In our study, although RT-PCR negativity was detected in all patients in the preoperative period, there were 59 $(10.7 \%)$ patients with RT-PCR positivity in endobronchial lavage samples taken in the early postoperative period. In a review by Patel et al. [18], it is mentioned that there are nearly $20 \%$ false positives in RT-PCR tests performed on nasopharyngeal swab samples.

With this study, we have achieved a new gain in the approach to cardiac surgery cases during the COVID-19 pandemic. The RT-PCR positivity detected in the endobronchial lavage samples in the postoperative period showed the necessity of developing a new algorithm in the approach to this particular patient group. In the light of this information, it is very important to take an endobronchial lavage sample in all medical situations requiring endotracheal intubation, regardless of the previous RT-PCR result, in terms of the follow-up and treatment process of the patient's clinic.

\section{Limitations}

The most important limitations of our study are that it is single-centered, retrospective, and the number of patients is low. More comprehensive publications with larger numbers of patients are needed to support existing data.

\section{CONCLUSION}

With the COVID-19 pandemic, serious changes have occurred in the practice of cardiac surgery, as in many medical conditions. Especially in the intensive care process after open heart surgery, endobronchial lavage samples taken from the endotracheal intubation tube are important in demonstrating the presence of COVID-19, which could not be detected previously in patients. In addition to being negative for PCR at least 2 times in routine preoperative preparations, obtaining endobronchial lavage samples for PCR testing from the intubation tube in the early postoperative period plays an important role in patient management.

\section{Authors' Contribution}

Study Conception: AKA, ŞY; Study Design: AKA, ŞY; Supervision: AKA, ŞY; Funding: AKA, ŞY; Materials: AKA, ŞY; Data Collection and/or Processing: AKA, ŞY; Statistical Analysis and/or Data Interpretation: AKA, ŞY; Literature Review: AKA, ŞY; Manuscript Preparation: AKA, ŞY and Critical Review: ŞY.

\section{Conflict of interest}

The authors disclosed no conflict of interest during the preparation or publication of this manuscript.

\section{Financing}

The authors disclosed that they did not receive any grant during conduction or writing of this study.

\section{REFERENCES}

1. Haft JW, Atluri P, Ailawadi G, Engelman DT, Grant MC, Hassan A, et al. Society of ThoracicSurgeons COVID-19 Task Force 
and the Work force for Adult Cardiac and Vascular Surgery. Adult cardiac surgery during the COVID-19 pandemic: a tiered patient triage guidance statement. Ann Thorac Surg 2020;110:697-700. 2. Niknam J, Rong LQ. Asymptomatic patients with coronavirus disease and cardiac surgery: when should you operate? J Card Surg 2020;35:2486-8.

3. Karaca U, Ata F, Yilmaz C, Balkaya AN, Onur T. Evaluation of anesthetic approaches to surgical patients during early COVID-19 pandemic. Eur Res J 2022;8:91-7.

4. Uysal A, Erturk E, Abacilar AF, Duman U, Dogan OF. The outcomes of patients incidentally confirmed with COVID-19 after cardiac surgery. Heart Surg Forum 2021;24:E940-6.

5. Barkhordari K, Khajavi MR, Bagheri J, Nikkhah S, Shirzad M, Barkhordari S, et al. Early respiratory outcomes following cardiac surgery in patients with COVID-19. J Card Surg 2020;35:2479-85.

6. Ata F, As AK, Engin M, Kat NK, Ata Y, Turk T. Can blood urea nitrogen-to-albumin ratio predict mortality in patients with moderate-to-severe COVID-19 pneumonia hospitalized in the intensive care unit? Rev Assoc Med Bras (1992) 2021;67:1421-6. 7. Al-Balas M, Al-Balas HI, Al-Balas H. Surgery during the COVID-19 pandemic: a comprehensive overview and perioperative care. Am J Surg 2020;219:903-6.

8. Wang W, Xu Y, Gao R, Lu R, Han K, Wu G, et al. Detection of SARS-CoV-2 in different types of clinical specimens. JAMA 2020;323:1843-4.

9. Rodriguez-Morales AJ, Cardona-Ospina JA, GutiérrezOcampo E, Villamizar-Peña R, Holguin-Rivera Y, Escalera-Antezana JP, et al., Latin American Network of Coronavirus Disease 2019-COVID-19 Research (LANCOVID-19). Clinical, laboratory and imaging features of COVID-19: a systematic review and meta-analysis. Travel Med Infect Dis 2020;34:101623.

10. Engin M, Aydın U, Eskici H, Ata Y, Türk T. Type 1 acute aortic dissection in the early period after COVID-19 infection. Cureus 2021;13:e13751.
11. Yates MT, Balmforth D, Lopez-Marco A, Uppal R, Oo AY. Outcomes of patients diagnosed with COVID-19 in the early postoperative period following cardiac surgery. Interact Cardiovasc Thorac Surg 2020;31:483-5.

12. As AK, Erdolu B, Duman B, Yazgan E, Eris C, Aydin U, et al. Can a modified-simplified pulmonary embolism severity index (m-sPESI) be used to predict the need for intensive care in hospitalized COVID-19 patients? J Thromb Thrombolysis 2021;52:759-65.

13. Guan WJ, Ni ZY, Hu Y, Liang WH, Ou CQ, He JX, et al.; China Medical Treatment Expert Group for Covid-19. Clinical characteristics of coronavirus disease 2019 in China. N Engl J Med 2020;382:1708-20.

14. Zhou F, Yu T, Du R, Fan G, Liu Y, Liu Z, et al. Clinical course and risk factors for mortality of adult in patients with COVID19 in Wuhan, China: a retrospective cohort study. Lancet 2020;395:1054-62.

15. Ad N, Luc JGY, Nguyen TC. COVID-19 North American Cardiac Surgery Survey Working Group. Cardiac surgery in North America and coronavirus disease 2019 (COVID-19): Regional variability in burden and impact. J Thorac Cardiovasc Surg 2021;162:893-903.e4.

16. Fattouch K, Corrao S, Augugliaro E, Minacapelli A, Nogara A, Zambelli G, et al. Cardiac surgery outcomes in patients with coronavirus disease 2019 (COVID-19): a case-series report. J Thorac Cardiovasc Surg 2022;163:1085-92.e3.

17. Lei S, Jiang F, Su W, Chen C, Chen J, Mei W, et al. Clinical characteristics and outcomes of patients undergoing surgeries during the incubation period of COVID-19 infection. EClinicalMedicine 2020;21:100331.

18. Patel V, Jimenez E, Cornwell L, Tran T, Paniagua D, Denktas AE, et al. Cardiac surgery during the coronavirus disease 2019 pandemic: perioperative considerations and triage recommendations. J Am Heart Assoc 2020;9:e017042. 